\title{
Shape optimization of a cementless hip stem
}

\author{
P.R. Fernandes ${ }^{1} \&$ R.B. Ruben ${ }^{2}$ \\ IIDMEC - Instituto Superior Técnico, Lisboa, Portugal \\ $2_{\text {Escola Superior de Tecnologia e Gestão, Leiria, Portugal }}$
}

\begin{abstract}
This work presents a development of a computational model for shape optimization of cementless hip stems. To improve the stability of the prosthesis, the optimization problem consists of the minimization of the relative displacements on bone/stem interface. The design variables are parameters that characterize the geometry of selected cross sections. These variables are subject to geometric constraints to ensure a clinically admissible shape. The stem/bone set is considered a structure in equilibrium with contact condition on interface. The contact formulation allows us to analyze different lengths of porous coating. The optimization problem is solved numerically by a steepest descent method where the sensitivity of objective function is computed using finite differences. The interface stresses and relative displacement is obtained solving the contact problem by the finite element method. Numerical examples are presented for a two-dimensional model of a hip stem, however the formulation is general and can be applied to the three-dimensional case. The two-dimensional model gives good indications about the relation between shape, porous coating and interface relative displacement.
\end{abstract}

\section{Introduction}

In last decades, hip prosthesis design, including cementless stems, has had a great development based on medical expertise and biomechanics research work. However, some clinical problems remained unsolved. Some of these problems are related with the stem stability. In fact, high bone/stem relative displacement and high stress level on interface can originate tight pain (see e. g. Herzwurm et al. [1]), avoid bone ingrowth (see e. g. Keaveny and Bartel [2]) and 


\section{Simulations in Biomedicine $V$}

consequently lead to a premature revision. The importance of the stem stability and interface modeling has been referred in recent numerical research, for instance in the work of Viceconte et al. [3] and Bernakiewick e Viceconti [4].

The factors mentioned above, namely the relative displacement and interface stress, depend directly on design parameters, such as stem geometry and porous coating extension, and an accurate selection of these parameters can improve stem stability. Although stem shape is strongly constrained by anatomic factors some numerical models for shape optimization have been proposed for stem design, such as the works of Huikes and Boeklagen [5] Katoozian and Davy [6] and Kowalczyk [7]. Furthermore, recent work on bone ingrowth prediction concludes that the stability can be related with the stem shape (Fernandes et al. [8]).

To answer the question: how is the prosthesis stability related with the stem shape? this work proposes a novel optimization model to determine the optimal shape of a cementless femoral component of a hip prosthesis. The problem is formulated by the minimization of relative displacements on bone/stem interface. A multiple load formulation allows us to consider different load cases corresponding to various types of daily life activities. The design variables are parameters that characterize successive stem sections. These design variables are subject to geometric constraints in order to obtain a clinically admissible shape. The set bone/stem is considered a structure in equilibrium with contact condition on interface. This contact formulation allow us to analyze different lengths of porous coating. The optimization problem is solved numerically by a steepest descent method where the sensitivity of objective function is computed using finite differences. The interface stresses and relative displacement are obtained solving the contact problem by the finite element method. Numerical examples are presented for a two-dimensional model of a hip stem. However, the formulation proposed is general and can be applied to the three-dimensional case.

\section{The Optimization Problem}

As mentioned above, the purpose of the optimization procedure is to obtain the optimal stem shape for a minimum of bone/stem relative displacement. Considering a displacement norm for objective function, and using a multiple load optimization criterion, the problem can be stated as,

$$
\min _{d_{l}} \sum_{P=1}^{N L}\left(\alpha^{P} \frac{1}{\left|\Gamma_{c}\right|} \int_{\Gamma_{c}}\left|\left(u_{t}^{r e l}\right)^{P}\right|^{2} d \Gamma\right)
$$

such that,

$$
\begin{aligned}
& \left(l_{i}\right)_{\min } \leq d_{i} \leq\left(l_{i}\right)_{\max } \\
& h\left(d_{i}\right) \leq 0
\end{aligned}
$$


where $N L$ is the number of applied load cases, $\alpha^{P}$ are the load weight factors satisfying $\sum_{P=1}^{N C} \alpha^{P}=1$ and $\left(u_{t}^{\text {rel }}\right)^{P}$ is the relative tangential displacement on contact surface $\Gamma_{c}$ for load case $P$. Design variables $d_{i}$ are parameters that characterize a given number of cross sections. These parameters are limited to lower and upper bounds, and subjected to a set of constraints (eqn 3 ) in order to ensure a clinically admissible shape. The relative displacement $\left(u_{t}^{\text {rel }}\right)^{P}$ for each load case $P$ is computed by the solution of the set of elasticity problems for two bodies in contact given by,

$$
\begin{aligned}
& \int_{\Omega} E_{i j k l} e_{i j}\left(\boldsymbol{u}^{P}\right) e_{k l}\left(\boldsymbol{v}^{P}\right) d \Omega-\int_{\Omega} b_{i}^{P} v_{i}^{P} d \Omega-\int_{\Gamma_{i}} t_{i}^{P} v_{i}^{P} d \Gamma+ \\
& \int_{\Gamma_{e}} \tau_{n}^{p}\left(v_{n}^{r e t}\right)^{p}+\tau_{t}^{p}\left(v_{t}^{r e t}\right)^{p} d \Gamma=0 \quad \forall v \text { admissible } \\
& \left\{\begin{array}{l}
\left(u_{n}^{r e l}\right)^{P}-g \leq 0 \\
\tau_{n}^{P} \geq 0 \\
\tau_{n}^{P}\left(\left(u_{n}^{r e l}\right)^{P}-g\right)=0 \\
\left|\tau_{t}^{P}\right| \leq \vartheta\left|\tau_{n}^{P}\right|\left\{\begin{array}{l}
\left|\tau_{t}^{P}\right|<\vartheta\left|\tau_{n}^{P}\right| \Rightarrow u_{t}^{r e l}=0 \\
\left|\tau_{t}^{P}\right|=\vartheta\left|\tau_{n}^{P}\right| \Rightarrow \exists \Lambda \geq 0: u_{t}^{r e l}=-\Lambda \tau_{t}^{P^{P}}
\end{array}\right.
\end{array}\right.
\end{aligned}
$$

In eqns (4) and (5), $E_{i j k l}$ is the elastic properties, $e_{i j}^{P}$ is the strain field and $v_{i}^{P}$ the set of virtual displacements. The last term of eqn (4) is the contribution of contact loads $\tau$, the subscripts $n$ and $t$ denotes normal and tangential directions, respectively. In eqn. (5) $g$ is the gap between the two bodies and $\vartheta$ is the friction coefficient.

\section{Definition of design variables and load cases}

The optimization problem formulated above is general, and can be solved for both two and three dimensional models. However, in this paper only results for the two-dimensional problem were obtained. In this case the design variables and load cases were defined as follows.

\subsection{Initial design and variables}

The initial stem shape was defined based on an existing stem design (Tri-Lock of 
DePuy) and six design variables were chosen as shown in figure 1. For each variable, lower and upper bound were defined in order to maintain the stem inside the bone, and, the following constraints,

$$
\begin{aligned}
& h_{1}=d_{6}-d_{5} \leq 0 \\
& h_{2}=d_{2} / 2-d_{5} \leq 0 \\
& h_{3}=d_{1}-d_{2} \leq 0
\end{aligned}
$$

were defined to ensure that the section size decrease from the proximal to distal part. The definition of these geometric constraint is very important to obtain a clinically admissible shape.

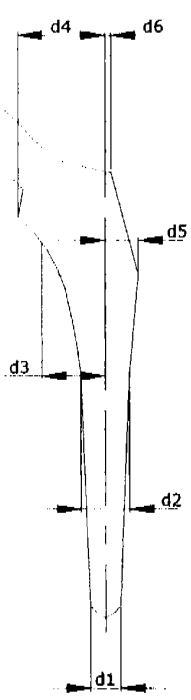

Figure 1: Definition of design variables on initial stem shape 1

\subsection{Load Cases}

To test the optimization model, three load cases were considered. The magnitude and direction of these load cases are summarized in table 1 and figure 2, respectively. These loads correspond to different daily activities, the first and the second correspond to walking and the third correspond to stair climbing (Kuiper [9]). It was also assumed that all threes cases have equal weight in the optimization process, i. e., $\alpha_{1}=\alpha_{2}=\alpha_{3}=1 / 3$. 


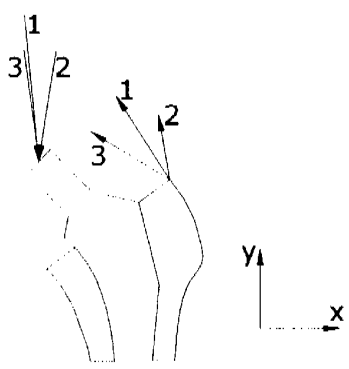

Figure 2. Load Cases

Table 1: Magnitude of load cases

\begin{tabular}{|c|c|c|c|}
\hline & & Fx(N) & Fy(N) \\
\hline load 1 & Fh & 224.4 & -2248.3 \\
& Fa & -818.6 & 1271.3 \\
\hline load 2 & Fh & -272.0 & -1693.2 \\
& Fa & -166.5 & 959.2 \\
\hline load 3 & Fh & 460.2 & -1717.4 \\
& Fa & -1280.0 & 728.3 \\
\hline
\end{tabular}

\section{Numerical model}

The optimization model given by eqns. (1-3) is solved using a steepest descent method with a penalty function (see for instance Luenberger [10]). In this method the update of design variables is given by

$$
\boldsymbol{d}_{k+1}=\boldsymbol{d}_{k}-\rho_{k} \alpha^{P} \nabla F^{P}\left(\boldsymbol{d}_{k}\right)
$$

where $\mathrm{F}$ is the penalty function defined, using the penalty factor $\mathrm{c}$ by,

$$
F^{P}=f^{p}+c / 2 \sum_{i=1}^{3} \max \left(0, h_{i}\right)^{2}
$$

for an objective funtion $f^{P}$ and a set of constraint $h_{\mathrm{i}}$. Note that the superscript $\mathbf{P}$ denotes the load case number and $\alpha^{P}$ is the respective weight.

In eqn. (7) $d_{k}$ is the vector of design variables at iteration $k$, $\boldsymbol{d}_{k}=\left\{\begin{array}{llllll}d_{1} & d_{2} & d_{3} & d_{4} & d_{5} & d_{6}\end{array}\right\}_{k}$, and $\rho_{k}$ is the step, which can be computed using Armijo's rule (Luenberger [10]). The gradient of objective function at each iteration is computed using forward finite differences.

The contact problem given by eqns. (6-7) to obtain the displacement field is solved using the commercial finite element code Abaqus [11].

Figure 3 presents the flow diagram for the optimization process. 


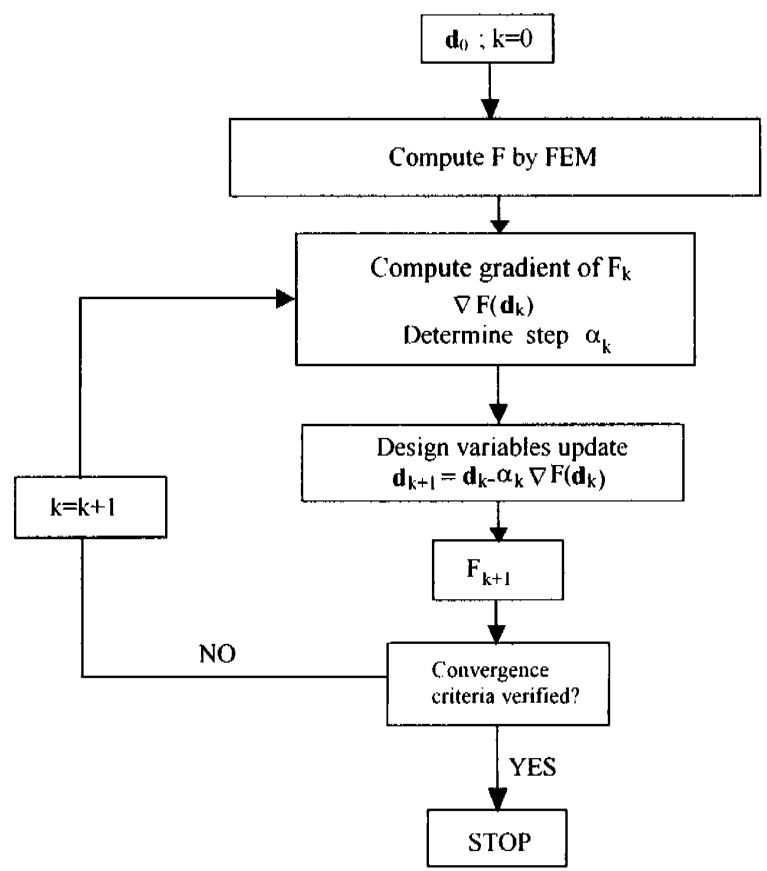

Figure 3: Flow diagram for the computational model

\section{Results}

The optimal stem shape were obtained for single load case situation, applying each load case individually, and then for all three cases together using the multiple load formulation. Since the contact formulation allows us to test different coating extensions, the problem was solved for four situations: uncoated, partially coated $1 / 4$, partially coated $1 / 2$ and totally coated. These situations are illustrated in figure 4 as well as the initial geometry. Interface conditions depend on coating extension. It is assumed contact without friction where the stem is uncoated and contact with friction where the coating exist. The value for the friction coefficient is 1.73, suggested by Keaveny and Bartel [2].

The model was discretized using plane stress linear finite elements. A side plate was considering simulating three-dimensional effects (see e. g. Jacobs et al. [12]). Thickness for bone, stem and side plate are shown in figure 5 and material properties are summarized in table 2 . Note that the numerical value for objective function was multiplied by $\lambda=10^{5}$ for the sake of numerical precision.

Figures 6 and 7 show the optimal configuration for load case 1 and 2 respectively, and figure 8 show the result for multiple load situation. Table 3 summarizes the numerical results for all cases. 


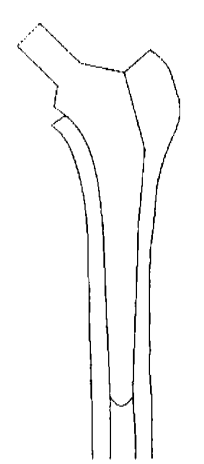

a)

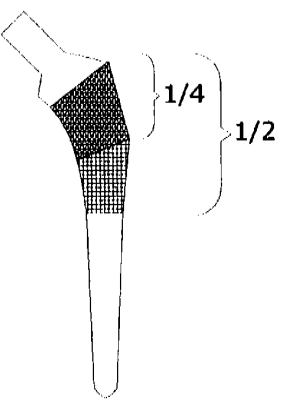

b)

Figure 4: a) Initial design for bone and prosthesis. b) Coating extension for partially coated cases.

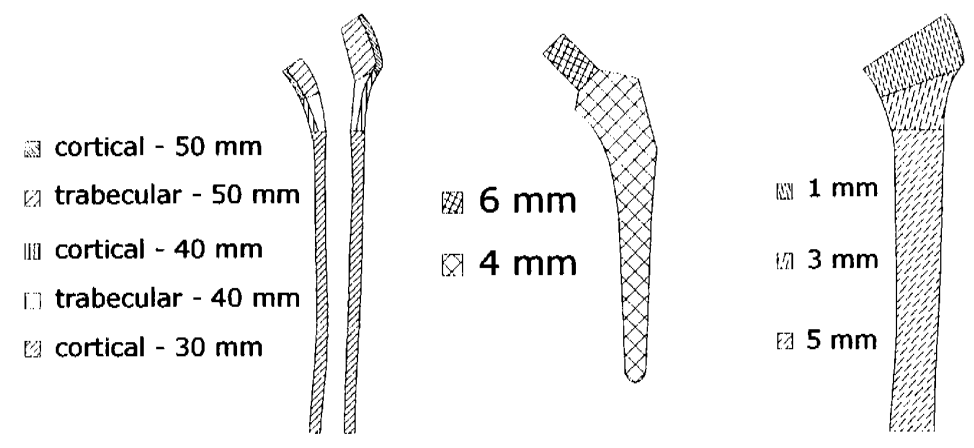

a)

b)

c)

Figure 5: Thickness for bone (a), stem (b) and side plate (c)

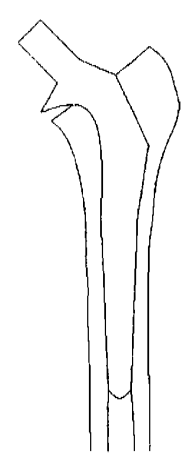

a)

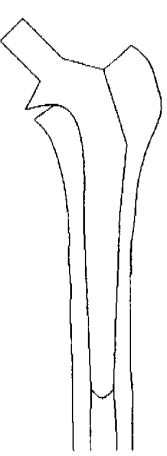

b)

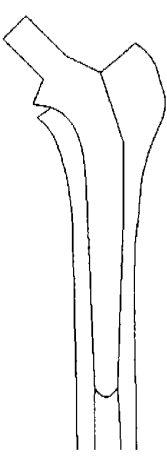

c)

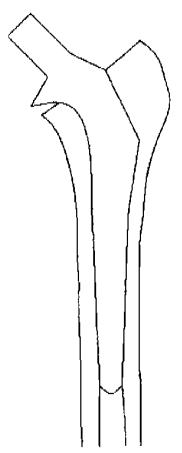

d)

Figure 6: Optimal shape for load case 1. a) totally coated b) partially coated $1 / 2$ c) partially coated $1 / 4$ d) uncoated stem 


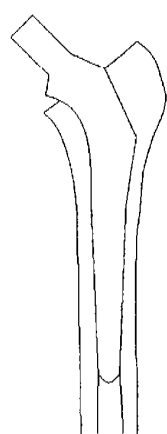

a)

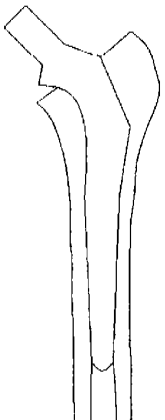

b)

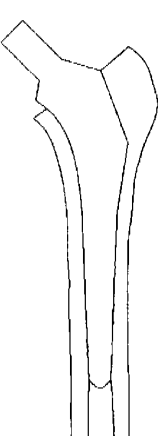

c)

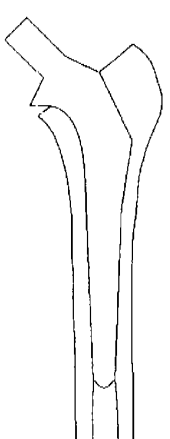

d)

Figure 7: Optimal shape for load case 2. a) totally coated b) partially coated $1 / 2$ c) partially coated $1 / 4$ d) uncoated stem

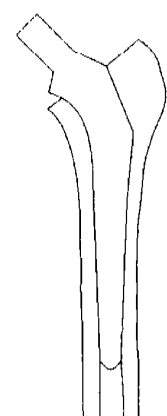

a)

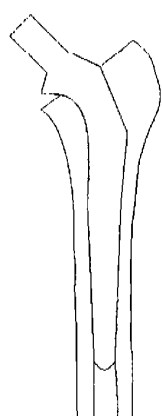

b)

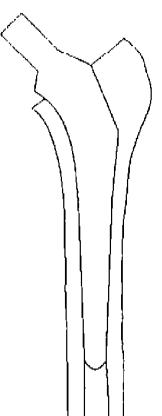

c)

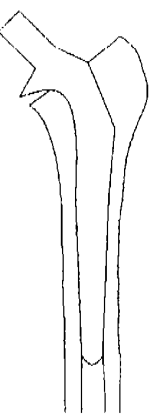

d)

Figure 8: Optimal shape for multiple load case. a) totally coated b) partially coated $1 / 2$ c) partially coated $1 / 4$ d) uncoated stem

Table 2: Material Properties

\begin{tabular}{|c|c|c|}
\hline & $\mathrm{E}(\mathrm{GPa})$ & $v$ \\
\hline Cortical Bone & 17 & 0.3 \\
\hline Trabecular Bone & 1 & 0.3 \\
\hline Stem & 115 & 0.3 \\
\hline
\end{tabular}

Table 3: Objective function values

\begin{tabular}{|c|c|c|c|}
\hline LOADS & COATING & $f$ initial & $f$ final \\
\hline \multirow{3}{*}{ Load case 1 } & uncoated & 16121.4 & 596.1 \\
& part. coated 1/4 & 97.4 & 87.6 \\
& part. coated 1/2 & 102.2 & 73.1 \\
& tot. coated & 72.3 & 65.7 \\
\hline \multirow{3}{*}{ Load case 2 } & uncoated & 5893.6 & 441.8 \\
& part. coated $1 / 4$ & 90.2 & 70.4 \\
& part. coated 1/2 & 209.6 & 106.4 \\
& tot. coated & 102.9 & 71.9 \\
\hline \multirow{3}{*}{ Multiple Load } & uncoated & 10792.9 & 2254.3 \\
& part. coated 1/4 & 97.0 & 94.0 \\
& part. coated 1/2 & 132.1 & 90.7 \\
& tot. coated & 102.9 & 82.7 \\
\hline
\end{tabular}




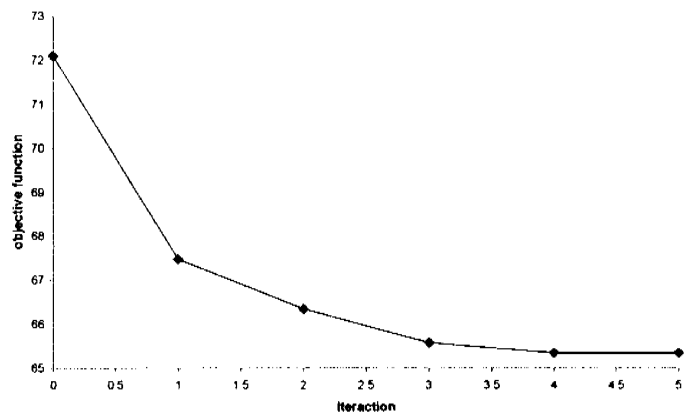

Figure 9: Convergence of objective function. Totally coated stem and load case 1 .

The optimization method proposed in this work seems to be very efficient. The convergence is illustrated in figure 10 , where the evolution of objective function is shown for load case 1 and totally coated stem.

The results show a formation of a support collar. This collar avoids subsidence of the stem. However, for the partially coated stem $(1 / 4)$ the collar is smaller than for the others situations. This fact can be explained because different coating extensions lead to different contact conditions. The stem with $1 / 4$ coating has larger displacement on medial side than on lateral side. On other hand, the displacement for the others situations is larger on lateral side. The collar reduces the displacement on lateral side. Thus, it is not necessary to form a big collar on the partially coated (1/4) stem. For the same reason the biggest collar is formed for the totally coated stem, since this stem has, at initial design, the higher contribution of the displacement on lateral side to the objective function.

\section{Final remarks}

This work presented an optimization process to find the shape of a hip stem in order to minimize the relative displacement on bone/stem interface. The set bone/stem is considered a structure in equilibrium with contact conditions. The optimization problem is formulated using a multiple load criteria. The formulation uses a contact model for bone/stem interface, which allows us to consider different extension of porous coating.

Results show a collar formation, which minimize the relative displacement. These results are consistent with others works (see e. g. Keaveny e Bartel [2]). Furthermore, these two-dimensional results give some good indications about the relation between stem shape, coating extension and interface relative displacement. In fact, the porous coating changes contact conditions and the collar size depends on these contact conditions, i. e., depends on the relation between the contribution to objective function of medial and lateral side relative displacement. 
Although the two-dimensional model gives good indications for prosthesis design, a three-dimensional model is needed for an accurate identification of stem shape. In fact, the twist play an important role on total relative displacement, and torsion can not be taken in account in a two-dimensional model (Keaveny and Bartel [2]). The model presented here is general and can be extended to the three-dimensional case with a correct parameterization of the stem cross sections. Also a multiple criteria formulation can be used to account simultaneously for interface stress and relative displacement.

\section{References}

[1] Herzwurm P. J., Simpson S. L, Duffin S., Oswald S. G. and Ebert, F. R., "Thigh Pain and Total Hip Arthroplasty", Clin. Orth. and Rel. Res., 336, pp. 156-161, (1997)

[2] Keaveny T. and Bartel D., "Efects of Porous Coating, With and Without Collar Support, On Early Relative Motion for a Cementless Hip Prosthesis", J. Biomechanics 26, pp.1355-1368 (1993)

[3] Viceconti M., Muccini R., Bernakiewicz M., Baleani M. and Cristofolini L., "Large-Sliding Contact Elements Accurately Predict Levels of Bone-Implant Micromotion Relevant to Osseointegration", $J$. Biomechanics, 33, pp. 1611-1618, (2000).

[4] Bernakiewick, M. E. and Viceconti M., "The Role of Parameter Identification in Finite Element Contact Analyses with Reference to Orthopaedic Biomechanics Applications", J. Biomechanics 35, pp.61-67 (2002)

[5] Huikes R. and Boeklagen R., "Mathematical Shape Optimization of Hip Prosthesis Design", J. Biomechanics, 22, 793-804 (1989).

[6] Katoozian H. and Davy D. T., "Three-Dimensional Shape Optimization of Femoral Components of Total Hip Prostheses" BED-Vol. 24, Bioengineering Conference ASME, pp.552-555 (1993)

[7] Kowalczyk P., "Design Optimization of Cementless Femoral Hip Prostheses Using Finite Element Analysis", J. Biomechanical Eng. Vol. 123, pp. 396-402, 2001.

[8] Fernandes P. R., Folgado J., Jacobs C. and Pellegrini V., "Bone Ingrowth on Cementless Femoral Stems-A comparison Study for Two Stem Geometries", $J$. Biomechanics, 34, Supp. 1, Proc. of the Biomechanica IV, pp. S18, (2001)

[9] Kuiper J. H., "Numerical Optimization of Artificial Joint Designs", Ph.D. Thesis, Katholieke Universiteit Nijmegen, (1993)

[10] Luenberger D. G., "Linear and Nonlinear Programming", Addison-Wesley Publishing Company, $2^{\text {a }}$ edição, (1989).

[11] ABAQUS, "User's Manual, Version 5.8", HKS, Inc, RI, USA. (1998).

[12] Jacobs C., Simo C., Beaupré G. and Carter D., "Adaptive Bone Remodelling Incorporating Simultaneous Density and Anisotropy Considerations", J. Biomechanics, 30, pp. 603-613, (1997) 